\title{
Localization and self-trapping in driven-dissipative polariton condensates
}

\author{
F. Bello and P. R. Eastham \\ School of Physics and CRANN, Trinity College Dublin, Dublin 2, Ireland \\ (Received 14 April 2017; revised manuscript received 6 June 2017; published 23 June 2017)
}

\begin{abstract}
We study driven-dissipative Bose-Einstein condensates in a two-mode Josephson system, such as a double-well potential, with asymmetrical pumping. We investigate nonlinear effects on the condensate populations and mode transitions. The generalized Gross-Pitaevskii equations are modified in order to treat pumping of only a single mode. We characterize the steady-state solutions in such a system as well as criteria for potential trapping of a condensate mode. There are many possible steady states, with different density and/or phase profiles. Transitions between different condensate modes can be induced by varying the parameters of the junction or the initial conditions, or by applying external fields.
\end{abstract}

DOI: 10.1103/PhysRevB.95.245312

\section{INTRODUCTION}

Structures which enable strong coupling between excitons and photons support hybrid light-matter quasiparticles known as exciton polaritons. As with cold atoms, above a certain density a population of polaritons may condense into a single mode and so form a Bose-Einstein condensate (BEC) [1,2]. Such macroscopic quantum coherence leads to a number of nonlinear dynamical effects, apparent in the condensate density, in trapped atomic BECs and superconducting Josephson junctions. These include Landau-Zener tunneling, population inversion, and self-trapping [3-9]. The realization of analogous effects for exciton-polariton condensates is important for optical communications technologies demanding ultrafast optical switches, long-range coherence, as well as picosecond quantum computing processes [10,11]. In this direction, advances in fabrication have led to successful demonstrations of Josephson oscillations as well as self-trapping in micropillar cavities [12,13]. The interplay between interactions and coherence, central to such phenomena, also has an important role in the formation of localized structures such as gap solitons $[14,15]$ and vortices $[16,17]$.

The polariton system differs from the atomic one because polaritons decay, with a lifetime which is typically on the order of a few picoseconds. The polariton condensate is thus a nonequilibrium steady state established by a balance of loss and gain, with the latter provided by stimulated scattering from an incoherent reservoir. This creates an interesting phenomenology, blending that of equilibrium condensates, which are dominated by interactions, with that of systems such as lasers, which are dominated by dissipation. For example, in two-mode systems the steady state can have density oscillations, which correspond to the a.c. Josephson effect [12,18-23]. Such oscillations also occur in the dissipationdominated regime, where they reflect the coexistence of two condensates of different frequencies, as in a multimode laser [19]. Indeed at the qualitative level many important phenomena, like gap solitons $[14,15]$, vortices [16], and condensate localization $[17,24,25]$, occur in both interaction-dominated and dissipation-dominated condensates.

In this paper we reexamine how the combination of gain, loss, and interactions affects the behavior of a polariton condensate in the simple two-state model of a Josephson junction $[18,19,21]$. The two states could correspond to neighboring local minima of a double-well potential in a coupled photonic molecule [13], or in a planar structure at a suitable point in a disorder potential [12]. They could, alternatively, correspond to two polarization states of a single trap mode [26]. Previous work on the incoherently pumped system has assumed that both modes are pumped [18-21,23]. Here we suppose that the single-particle modes are approximately symmetrical, but only one is pumped. We find that this model has a rich phase diagram, with many different steady states. These include states where the density is symmetrical in the two wells, as well as states where the condensate becomes trapped in the pumped well. The appearance of asymmetrical states is similar to the phenomenon of macroscopic quantum self-trapping, as previously analyzed for polaritons without gain and loss [26], and for resonant pumping $[27,28]$. In these cases, however, the asymmetry is caused by strong interactions, which prevent a complete transfer of population between the wells. We show that this requirement can be relaxed in the nonequilibrium case, and that the nonlinear gain, as well as interactions, can induce a form of self-trapping.

Condensation in photonic molecules formed from two micropillar resonators with asymmetrical pumping has been investigated by Galbiati et al., [29]. However, although in this experiment the photonic molecule was symmetrical, the pumping led to a large detuning between the states of each resonator. This was caused by the interaction with the high energy excitons created by the pump. In this large detuning regime the Josephson coupling has a negligible effect, and our calculations do not apply. Our predictions could, instead, be tested by using asymmetrical photonic molecules, constructed such that the single-particle states come into resonance when they are blueshifted by the interaction with the exciton reservoir.

\section{MODEL}

We model the condensate at the mean-field level, using the complex Ginzburg-Landau equation for the macroscopic wave function [16]. This generalizes the Gross-Pitaevskii equation (or nonlinear Schrödinger equation) to include the loss of polaritons due to their decay into photons and the gain due to scattering into the condensate from exciton states at high energies. In the case of a polariton condensate these processes can be treated using local terms, because the exciton mass is very large and gain diffusion is negligible. 
We consider the complex Ginzburg-Landau theory for a double-well potential in the two-mode limit, where the polaritons can occupy the ground states of a well on the left or the right of a barrier. The amplitudes of the macroscopic wave functions $\Psi_{l, r}$ for the left (l) and right (r) wells thus obey $(\hbar=1)$ :

$$
\begin{gathered}
i \frac{d \Psi_{l}}{d t}=\frac{\epsilon}{2} \Psi_{l}-J \Psi_{r}+U_{l}\left|\Psi_{l}\right|^{2} \Psi_{l}+i\left[g-\Gamma\left|\Psi_{l}\right|^{2}\right] \Psi_{l}, \\
i \frac{d \Psi_{r}}{d t}=-\frac{\epsilon}{2} \Psi_{r}-J \Psi_{l}+U_{r}\left|\Psi_{r}\right|^{2} \Psi_{r}-i \gamma \Psi_{r} .
\end{gathered}
$$

Here $\epsilon$ is the energy difference between the wells, including the mean-field interaction with the exciton reservoir [29]. $J$ is the tunneling strength, and $U_{l}$ and $U_{r}$ are the matrix elements of the polariton-polariton interaction [30] in the localized basis. The final two terms in Eq. (1) are the net linear gain, with coefficient $g$, and the lowest order nonlinear gain, with coefficient $\Gamma$. For the isolated well condensation occurs for positive $g$. In this case the linear gain term generates an exponential growth that is stabilized by the nonlinear term. The balance of these terms establishes a steady-state condensate with density $n_{0}=g / \Gamma$. We assume that the right well is either unpumped or pumped below threshold so that the amplitude there decays with rate $\gamma$.

It is convenient to introduce dimensionless fields by replacing $\Psi_{r(l)} \rightarrow \Psi_{r(l)} \sqrt{n_{0}}$, so that $n_{0}$ becomes the unit of density. We take the corresponding blueshift $U_{l} n_{0}$ to be the unit of energy and factor out an overall oscillation and phase difference between the modes by setting $\Psi_{r(l)}=$ $e^{-i \omega t} \psi_{r(l)} e^{(-) i \theta / 2}$. Thus we obtain a dimensionless form for the equations of motion

$$
\begin{gathered}
i \dot{\psi}_{l}=E_{l} \psi_{l}-J \psi_{r} e^{i \theta}+i g\left[1-\left|\psi_{l}\right|^{2}\right] \psi_{l}, \\
i \dot{\psi}_{r}=E_{r} \psi_{r}-J \psi_{l} e^{-i \theta}-i \gamma \psi_{r},
\end{gathered}
$$

where the energies include the mean-field shifts due to the interactions within the condensate,

$$
\begin{aligned}
& E_{l}=\frac{\epsilon}{2}-\omega+n_{l}, \\
& E_{r}=-\frac{\epsilon}{2}-\omega+\frac{U_{r}}{U_{l}} n_{r},
\end{aligned}
$$

and the populations are defined as $n_{l(r)}=\left|\psi_{l(r)}\right|^{2}$. Here $J, \epsilon, \gamma, \omega, g$ are measured in units of $U_{l} n_{0}$. The dimensionless $g$ is $g / U_{l} n_{0}=\Gamma / U_{l}$ and is called $\alpha$ in Ref. [31]. We note that although this parameter is a dimensionless pump rate it is independent of the original, dimensionful, pump rate in the model. It is better understood as a nonequilibrium control parameter, which is zero in equilibrium, and large when the dissipative nonlinearity dominates over the interactions, $\Gamma \gg U$, as in a conventional laser.

Another theory of the driven-dissipative condensate is used in Refs. [20,32-34]. In this theory the growth of the condensate, which is due to in-scattering from a reservoir of high-energy excitons created by the pump, is modeled by a linear gain term. Although the nonlinear gain is not explicit it is nonetheless present, because the linear gain coefficient depends on the occupation of the exciton reservoir. Solving for this reservoir occupation on timescales for which it is time independent gives a gain coefficient $g(n)$, which is a function of the condensate occupation. Expanding this as a power series and retaining the first two terms, $g(n)=g-\Gamma n \ldots$, gives the form in Eq. (1). Note that above threshold one must include at least the first two terms otherwise the solution is unphysical; below threshold the second can safely be neglected (as in Ref. [35]).

As well as the interaction within the condensate $U$, there are also interactions between the condensate and the population of high-energy excitons created by the pump [29], which give rise to energy shifts that are included in the definition of $\epsilon$. Thus in order to explore the near-resonant case $\epsilon=0$ discussed below one should consider a system where the bare modes are off-resonance, so that they are brought into resonance under asymmetrical pumping. We note that in the off-resonance case relaxation processes provide the dominant coupling between the wells. These are not present in our theory; we consider the case, near-resonance, where the Josephson coupling dominates.

We have also neglected, in writing Eqs. (1) and (2), the spin of the polariton [36]. The spin projection of the polariton takes two values, corresponding to right and left circular polarization states. However, condensates typically show a high degree of polarization, generally linear polarization in a direction determined by the crystal. In such cases only a single polarization state is relevant, and the model can be used to describe the spatial structure of the condensate, i.e., the extrinsic Josephson effects. The model could be generalized to include both polarizations, making the polariton wave function in each well a spinor. In this case one can have, as well as the extrinsic effects, intrinsic Josephson effects connected to the tunneling between the two polarization states. These have been studied in a model without gain and loss [26] and for the case of symmetrical pumping [20,21].

\section{STEADY-STATE ANALYSIS}

We now derive the phase diagram of the double-well system, examining the steady-state solutions of Eqs. (3) and (4), and their stability. For simplicity we suppose that the detuning $\epsilon$ is zero, and the interaction strengths are identical, $U_{l}=U_{r}=U$. We consider steady states with a single characteristic frequency $\omega$, which corresponds to the chemical potential in the equilibrium case. This is justified by our numerical results, in which the steady states are always of this class; we have not observed steady states with a.c. Josephson oscillations. Setting the time derivatives in Eqs. (3) and (4) to zero, multiplying by $\psi_{l}$ and $\psi_{r}$, respectively, and taking real and imaginary parts, gives:

$$
\begin{gathered}
g\left(1-n_{l}\right) n_{l}=J \sqrt{n_{l} n_{r}} \sin (\theta) \\
\gamma n_{r}=J \sqrt{n_{l} n_{r}} \sin (\theta) \\
E_{l} n_{l}=\left(n_{l}-\omega\right) n_{l}=J \sqrt{n_{l} n_{r}} \cos (\theta) \\
E_{r} n_{r}=\left(n_{r}-\omega\right) n_{r}=J \sqrt{n_{l} n_{r}} \cos (\theta) .
\end{gathered}
$$

Equations (6) and (7) describe the current flows in the double well. The term on the left of Eq. (6) describes the flow of polaritons from the reservoir to the left well, which is 
nonzero if the density there deviates from the value $n_{l}=1$ at which the linear gain is reduced to zero by the gain depletion. In the steady state this current must flow as a Josephson current into the right well, which is given by the term on the right-hand side of Eq. (6). This Josephson current in turn accounts for the loss from the right well, as described by Eq. (7). Equations (8) and (9) are the associated pressure balance (quantum Bernoulli) equations, which state that in the steady state the two populations must be in mechanical equilibrium through the Josephson coupling.

Noting the equality of the right-hand sides of Eqs. (8) and (9) we have

$$
\left(n_{l}-\omega\right) n_{l}=\left(n_{r}-\omega\right) n_{r} .
$$

This has a solution corresponding to the normal state, $n_{l}=$ $n_{r}=0$. It has two further solutions, one with $n_{l}=n_{r}$, and one with $n_{l} \neq n_{r}$. Thus there are two classes of condensed steady state: one in which the density is symmetrical, as expected from the linear eigenstates of the symmetrical double well, and one in which it is asymmetrical, due to the asymmetry of the pumping.

\section{A. Delocalized solutions}

We consider first the solution in which the condensate density is equal in the two wells, $n_{l}=n_{r}$. In this case we obtain, from Eqs. (6) and (7),

$$
n_{l}=n_{r}=1-\frac{\gamma}{g} \text {. }
$$

This gives a phase boundary $\gamma=g$ shown in Fig. 1, which separates the normal state (labeled $\mathrm{N}$ ), from the symmetricaldensity condensed states (labeled S0 and $\mathrm{S} \pi$ ). This phase

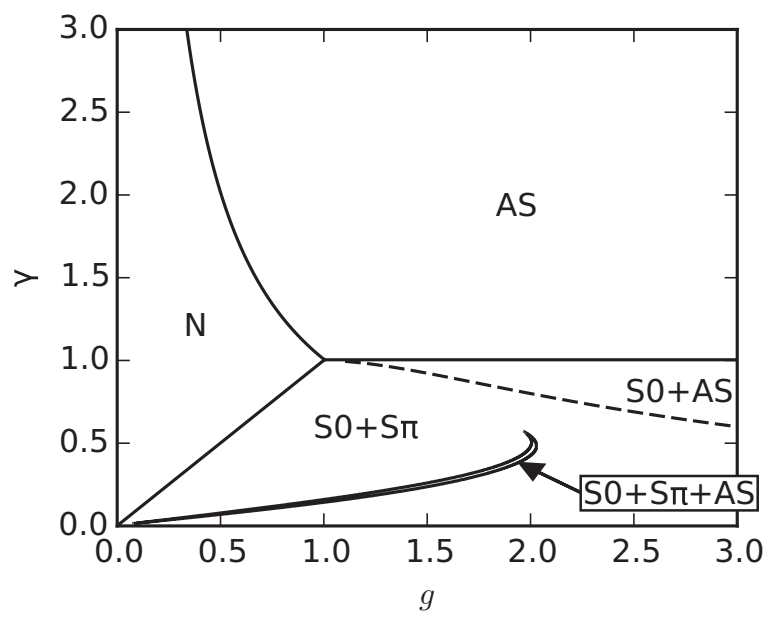

FIG. 1. Phase diagram for the asymmetrically-pumped doublewell condensate, as a function of the dimensionless pump rate $g$ for the left well and loss rate $\gamma$ for the right well. The tunneling strength $J=1$, and all energies and rates are measured in units of the mean-field energy shift of the left well. The solid and dashed lines mark phase boundaries (see text). The labels indicate the states present in each region: the normal state with no condensate $(\mathrm{N})$, two condensed states with equal densities in the two wells but different relative phases (S0,S $\pi$ ), and a condensed state with unequal densities in the two wells (AS). boundary is simply the requirement that the pumping must be sufficient to overcome the losses in the unpumped well. A second phase boundary follows on noting that, from Eq. (7), the phase difference between the wells is given by

$$
\gamma=J \sin (\theta) \text {. }
$$

Thus the solution requires $\gamma<J$. With increasing $\gamma$ there is a phase transition, at which this type of condensate breaks down because the interwell current imposed by the gain and loss exceeds the Josephson critical current.

As noted in the introduction, previous work on the incoherently-pumped junction has focused on the case of symmetrical pumping [18-21]. As we find here, in some parameter regimes there are d.c. Josepshon-like states, in which the tunneling leads to a single condensate, and there is a fixed phase relationship between the wells. Polarization splittings give rise, similarly, to an intrinsic Josephson effect, fixing the polarization direction of each mode and leading to polarization locking in extended systems [20,21]. In general such d.c. Josephson states break down if the interwell (for the extrinsic Josephson effect) or interpolarization (for the intrinsic effect) currents exceed the Josephson critical current. For symmetrical pumping, this leads to a transition to an a.c. Josephson-like state $[18,19,21]$. Here we find instead that it drives a localization transition, forming a d.c. Josephson-like state with an imbalanced density.

In general Eq. (12) has two solutions, so that there can be two symmetrical density condensates, with interwell phase differences in $(0, \pi / 2)$ or $(\pi / 2, \pi)$, respectively. The condensate energy $\omega$ for these two solutions follows from Eq. (8),

$$
\omega=n \mp \sqrt{J^{2}-\gamma^{2}} \text {. }
$$

For small $\gamma / J$ one solution has $\theta \approx 0$, and one has $\theta \approx \pi$. These values correspond to those expected from the linear regime [19], i.e., condensation in the symmetrical or antisymmetrical orbital. The condensate energy, Eq. (13), is then the expected single-particle energy shifted by the mean-field interaction. Increasing $\gamma$ introduces interwell currents, shifting the phase difference away from the limiting values of 0 and $\pi$ and increasing (decreasing) the energy of the ground-state (excited-state) condensate.

In conventional equilibrium Josephson junctions, where $J>0$, the $\pi$ state is unstable, since it corresponds to an energy maximum. However, in the driven-dissipative condensate both phase states can be stable. The stability can be determined by linearizing Eqs. (3) and (4) about the steady-state solution. The details are given in the Appendix, and the results have been incorporated on the phase diagrams shown in Figs. 1 and 2. We see that both phase states are stable near the onset of condensation at $\gamma=g$, but as $g$ increases the higher-energy solution, labeled $\mathrm{S} \pi$, eventually becomes unstable. For small $J$, corresponding to a condensate in which the interactions dominate over the tunneling, the $\mathrm{S} \pi$ state is restricted to a small region near the onset of condensation (see Fig. 2), whereas for larger $J$ there is a large region where both solutions are stable (see Fig. 1). Thus we predict that polariton condensates can realize two phase states in a single junction, so long as the interactions are not too strong compared with the tunneling. 


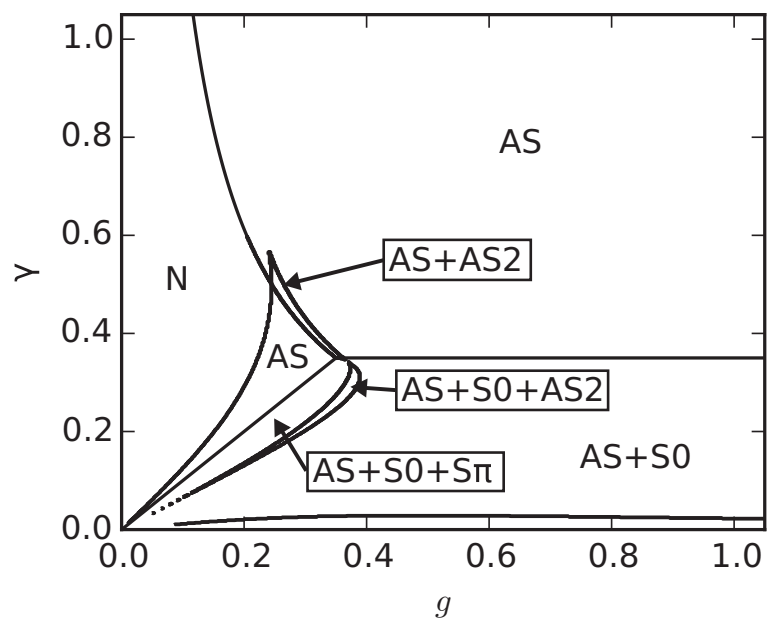

FIG. 2. Phase diagram for the asymmetrically-pumped doublewell condensate with tunneling strength $J=0.35$, for comparison with Fig. 1. The labels indicate the states present in each region: the normal state with no condensate $(\mathrm{N})$, two condensed states with equal densities in the two wells but different relative phases ( $\mathrm{S} 0, \mathrm{~S} \pi$ ), and two condensed states with unequal densities in the two wells (AS, AS2).

\section{B. Asymmetrical-density solutions}

The second class of solution has $n_{l} \neq n_{r}$ and corresponds to the situation in which the condensate density occupies predominantly one of the two wells. For such a localized or trapped solution Eq. (10) gives the condensate frequency

$$
\omega=n_{l}+n_{r} .
$$

This corresponds to the mean-field energy shift for the total occupation of the wells and contrasts with the corresponding result for the symmetrical-density solutions, Eq. (13). The densities may be determined by equating the left-hand sides of Eqs. (6) and (7), giving

$$
n_{r}=\frac{g}{\gamma}\left(1-n_{l}\right) n_{l} .
$$

Using this result, along with Eq. (8), in Eq. (6), leads to a cubic for the left-well density

$$
n_{l}^{3}-n_{l}^{2}+\gamma^{2} n_{l}+\gamma^{2}\left(\frac{J^{2}}{g \gamma}-1\right)=0 .
$$

The interwell phase $\theta$ is then determined by inserting the calculated densities and condensate frequency into Eqs. (6)-(9), so completing the solution. Note that this fully determines the interwell phase for each solution of Eq. (16) (up to the trivial addition of multiples of $2 \pi$ ); in contrast to the symmetrical-density case there is only one steady state for each $n_{l} \neq n_{r}$.

The cubic Eq. (16) implies that the asymmetrical condensate can appear continuously or at a first-order transition. The potential continuous transitions correspond to a real root becoming physical when the occupation $n_{l}$ crosses zero from below, which we see from the final two terms in Eq. (16) occurs at

$$
\gamma=J^{2} / g
$$

This result is plotted on the phase diagram in Fig. 1: It gives the continuous transition between the normal state and an asymmetrical-density condensate in the top-left quadrant. To the right of this curve there is a real-valued and positive solution for the condensate density. The potential first-order transitions occur when a pair of complex roots become real. The discriminant of the cubic, Eq. (16), is a quadratic in the pump parameter $g$, giving a pair of potential first-order phase boundaries, which meet at a critical point which is at $\gamma=1 / \sqrt{3}$. We will discuss these results further in the next subsection.

\section{Phase diagram}

To complete the derivation of the phase diagram we combine the results of the previous subsections with a linear stability analysis of the steady states, whose details are given in the Appendix. The results are shown in Figs. 1 and 2, in which the curves are the phase boundaries at which stable, physical steady-state solutions appear or disappear.

We discuss first Fig. 1, which corresponds to $J=1$, so that the tunneling strength is equal to the interaction energy scale of the left well. We see that for $\gamma<J=1$ there is a continuous transition, crossed as the parameter $g$ increases from zero, from the normal state to a symmetrical-density condensate. The phase boundary is $\gamma=g$, as predicted by Eq. (11). For larger $\gamma>J=1$ the transition is instead from the normal state to an asymmetrical-density condensate and follows the boundary given by Eq. (17). The continuation of this curve into the region $\gamma<J$ does not correspond to a phase boundary: The solution which crosses zero density with increasing $g$ or $\gamma$ in this region, and so becomes physical, does not become stable. It becomes stable along the phase boundary shown as the dashed curve, where also the higher energy of the two symmetrical-density solutions, labeled $\mathrm{S} \pi$, becomes unstable. Above this is another, horizontal, phase boundary at $\gamma=J$, as predicted by Eq. (12), at which the remaining symmetrical-density solution disappears.

As noted above, further asymmetrical-density solutions are possible when the discriminant of Eq. (16) is positive, so that there are three real roots for the density. Considering also the stability of these solutions we find, for this value of $J$, the small curved region shown in the figure, lying inside the symmetrical-density regime. In this curved region there is an additional asymmetrical density solution, which appears and then disappears with increasing $g$ at fixed $\gamma$ at the two first-order phase boundaries shown. The phase boundary for smaller $g$ corresponds to the discriminant of Eq. (16) reaching zero, and that for larger $g$ corresponds to the state becoming unstable.

For comparison, we show in Fig. 2 the phase diagram for $J=0.35$, corresponding to an interaction strength approximately three times the tunneling strength. The ranges of the axes have been adjusted so that the ground-state symmetric-density condensate, S0, occupies the same area in the two figures. As noted above, the other symmetric-density state is suppressed by the strong interactions and now occurs only over a small regime just to the right of the boundary $\gamma=g$. The strong interactions instead favor the asymmetric states: There is now a significant area of the phase diagram 


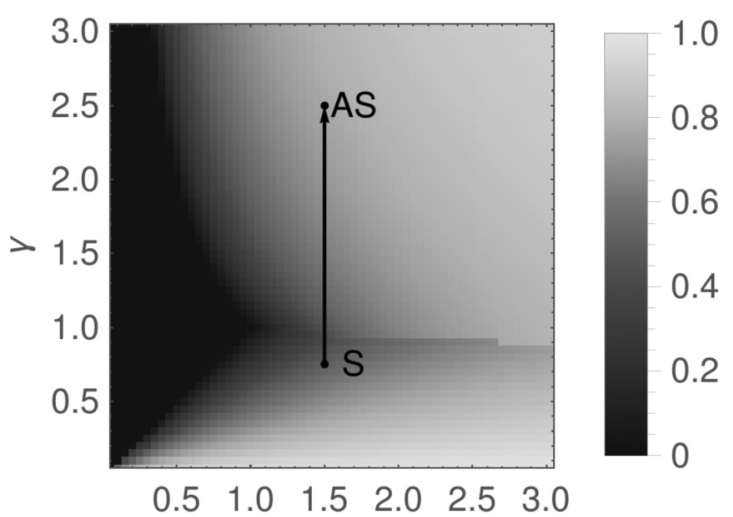

g

FIG. 3. Numerically calculated left well population for $J=1$ showing the three distinct states N, S, and AS predicted in Fig. 1. The time evolution is shown in Fig. 5 for the highlighted points at $g=1.5$ for the modes $\mathrm{S}$ and AS corresponding to $\gamma=0.75,2.5$, respectively.

where an asymmetric state coexists with the SO state, and there are small regions where two asymmetric solutions coexist. The first-order transition at which an asymmetric state appears with increasing $g$ now lies partially inside the normal region, and this state survives over a much wider range of parameters.

\section{NUMERICAL RESULTS}

In this section we numerically solve Eqs. (1) and (2) so as to study the steady states and transient behavior of the on-resonance double-well system. We determine the steadystate densities by evolving from an initial state chosen to be $\psi_{l}(0)=1, \psi_{r}(0)=0$. We take the dimensionless $J=1$ for comparison with Fig. 1. The three distinct regimes, corresponding to the normal state $(\mathrm{N})$, condensation with equal densities in the wells (S), and condensation with unequal densities (AS), are apparent in Figs. 3 and 4, which show the populations of the left and right wells after the transient. These results are clearly consistent with the phase boundaries

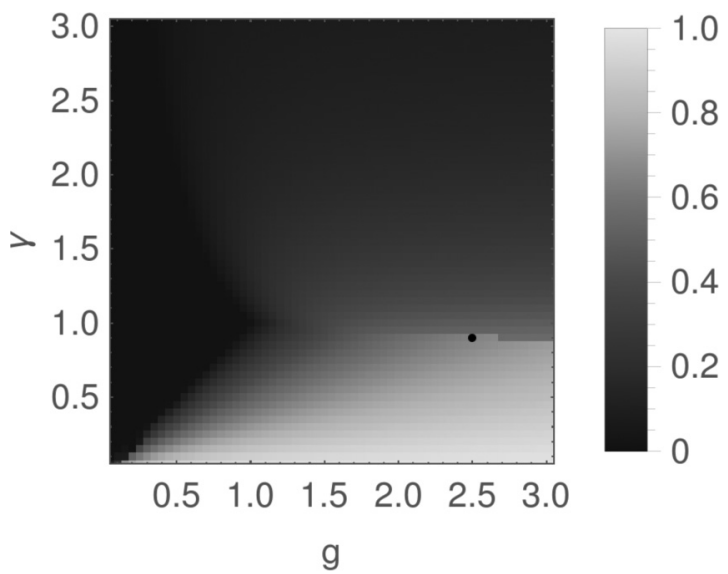

FIG. 4. Numerically calculated right well population for $J=1$ showing the three distinct states N, S, and AS predicted in Fig. 1. The time-dependent solution at $\gamma=0.9$ and $g=2.5$ is shown in Fig. 6 in order to demonstrate control over condensate mode as a function of initial conditions. obtained above. In particular we see the transition between the normal state and an asymmetrical condensate at $\gamma=J^{2} / g$ and that between the normal state and a symmetrical condensate at $\gamma=g$. There is also a discontinuity corresponding to switching between these solutions, close to the line $\gamma=J$. Above this line, as argued previously, the symmetrical solution breaks down, because the Josephson current is unable to compensate for the unbalanced pumping. As shown in Fig. 1, the asymmetric solution is in fact stable below this line (i.e., for smaller $\gamma$ ) giving a region of parameter space in which both steady states are stable. In this parameter regime the steady state is selected by the initial conditions for the condensate mode. This is consistent with the numerical results, where the discontinuity lies in the region of phase coexistence.

The appearance of imbalanced densities for a condensate in a double-well potential resembles the self-trapping of equilibrium condensates [3]. However, self-trapping in equilibrium is an effect of interactions and occurs only when the interaction strength dominates over the tunneling $U n \gg J$. In contrast we see that in nonequilibrium condensates asymmetrical densities can be caused by an inhomogeneous pumping. Since the phase boundary $\gamma=J$ is independent of the interaction strength, the transition to asymmetrical condensation can be induced by increasing the dissipation $\gamma$, even when the interactions $U$ are negligible. The effect is instead driven by the dissipative nonlinearity $\Gamma$.

As an example of trapping by dissipation we follow the trajectory marked with the arrow in Fig. 3, increasing the dissipation through the transition at fixed $g$. In practice, this could be achieved by reducing the pumping: recall that the dimensionless decay rate is the physical decay rate in units of the mean-field energy shift. It might instead be achieved by using an additional weak pumping of the right well to manipulate its decay rate (it may also be necessary to manipulate the bare detuning, to offset the change in reservoirinduced blueshift). As the dimensionless loss in the right well increases the steady-state transitions from a symmetrical to an asymmetrical density profile. Thus the output of the unpumped well can be switched by controlling its loss. The dynamics of the populations at the two extreme values of $\gamma$ highlighted in Fig. 3 is shown in Fig. 5. For small $\gamma$ the population imbalance

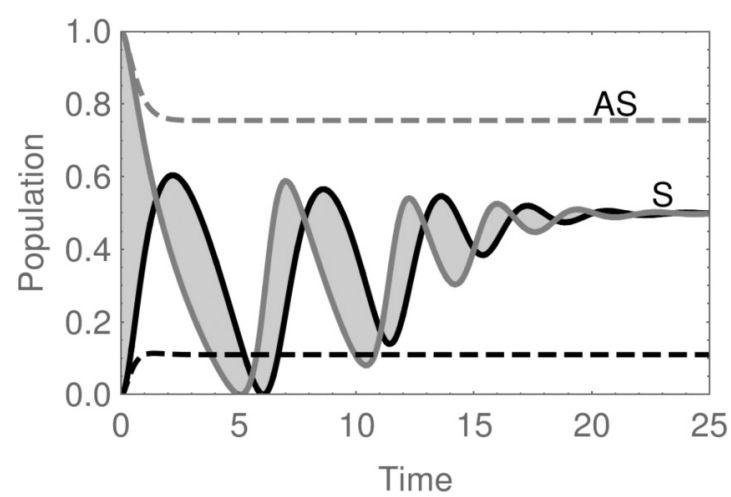

FIG. 5. Time dependent populations for the left (gray) and right (black) wells for the parameters corresponding to the points highlighted in Fig. 3. As loss $(\gamma)$ increases the steady state switches from a symmetric density mode ( $\mathrm{S}$, solid curves) to an asymmetric one (AS, dashed curves). The initial state is taken to be $n_{l}=1, n_{r}=0$. 


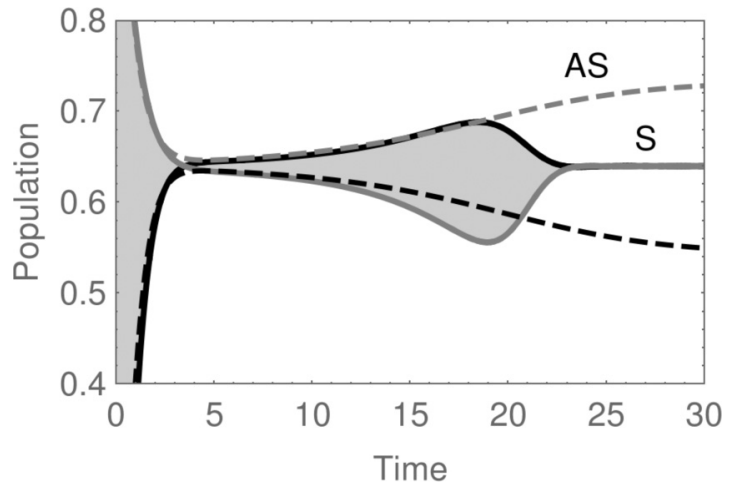

FIG. 6. Time dependent populations for the left (gray) and right (black) wells in the two-state region of Fig. 1: $\gamma=0.9$ and $g=2.5$. Depending on the initial conditions either the symmetric density mode (S) or the asymmetric one (AS) is reached. The initial states have $n_{l}=1$ (S, solid curves) or $n_{l}=2$ (AS, dashed curves), and $n_{r}=0$.

introduced in the initial condition oscillates between the two wells, and these oscillations decay towards a steady state with equal densities. Close to that steady state these oscillations can be interpreted as due to the Josephson plasmon of the junction, which is damped by the gain and loss; its frequency and decay rate follows from the stability analysis in the Appendix. With increasing $\gamma$ the oscillations disappear, and the condensate rapidly reaches a steady state which is trapped in the left well. This dissipative method differs from previous self-trapping mechanisms which use the interaction energy $U n$ to control the trapping of condensates [37].

Self-trapping could also be induced by varying the initial conditions for those values of $\gamma$ and $g$ where both symmetrical and asymmetrical steady states are stable. In these regimes (similarly to equilibrium self-trapping [4]) the initial conditions for the condensate mode determine whether the steady state has a symmetrical or asymmetrical density. A specific example given in Fig. 6 demonstrates this for the highlighted point in Fig. 4. For the corresponding values of $\gamma$ and $g$, we look at two different initial conditions for which the left well is altered from $\psi_{l}(0)=1$ to a value further above threshold $\psi_{l}(0)=2$. In the latter case, the Josephson current does not become sufficient to populate the wells symmetrically, and we see the steady-state mode change from the symmetric to asymmetric one. One can also switch between these steady states, as well as the different phase states of the symmetrical condensate, by driving the wells externally. This could be used to implement optical switches and memories, with the desirable feature that the output of one switch (the intensity from one of the wells) can form the input of another. The possibility of manipulating the initial conditions so as to induce self-trapping for polariton condensates has already been shown experimentally in Ref. [13], considering self-trapping as a transient phenomenon in the absence of gain. The present work shows how such concepts can be extended to apply to the steady states of driven-dissipative condensates.

In experiments the initial conditions for a driven-dissipative condensate might be controlled by using a resonant seed pulse to prepare the initial state. If the pump is then rapidly switched on then the state selected will be determined by these initial conditions. An alternative possibility would be to slowly increase the pumping, in which case we expect the system to adiabatically follow a particular state in the phase diagram. Large changes in pumping, however, would require a corresponding change in the bare detuning, in order to maintain the modes near resonance. In practice it would not be possible to keep the modes exactly resonant, as assumed above. However, we have checked numerically that this is not essential. We find that for small nonzero detunings the transition from a symmetrical to asymmetrical density mode survives, although it becomes one from a weakly to a strongly asymmetrical mode.

\section{SUMMARY}

To summarize, we have studied the steady states and dynamics of a two-mode polariton condensate, such as the Josephson junction in a double-well potential, for the case where one mode is pumped. We have shown that such asymmetrical pumping provides a mechanism by which condensates can become localized in one well which, in contrast to conventional self-trapping, does not rely on strong interactions, being caused instead by nonlinear gain. This will help open up the study of condensate localization and self-trapping in driven-dissipative condensates, which we predict can occur in both strongly and weakly interacting regimes. As we have shown, a simple two-mode polariton condensate supports a variety of coherent steady states, distinguished by both their density and phase profiles. It would be interesting to extend our work to systems such as lattices of junctions and so explore the nonlinear dynamics of spatially extended driven-dissipative condensates. Another interesting direction would be to consider the use of time-dependent parameters to control the trapping $[38,39]$. The possibility of realizing multiple steady states in a two-mode condensate, and switching between these states with applied fields, may be useful for optical switches and memories.

\section{ACKNOWLEDGMENTS}

P.R.E. acknowledges support from Science Foundation Ireland (SFI) Grant No. 15/IACA/3402. F.B. acknowledges support from SFI Grant No. 15/IFB/3317 and the SFI funded center CONNECT Grant No. 12/RC/2276.

\section{APPENDIX: STABILITY ANALYSIS}

In the main text we present results for the stability of the steady states of Eqs. (3) and (4). This is determined by setting $\psi_{l(r)}(t)=\psi_{l(r)}^{0}+\delta_{l(r)}(t)$, where $\psi_{l(r)}^{0}$ are the steadystate fields. The equations-of-motion, to first order in $\delta$, can be written in the block form

$$
i \frac{d}{d t}\left(\begin{array}{c}
\eta \\
\eta^{*}
\end{array}\right)=\left(\begin{array}{cc}
A & B \\
-B^{*} & -A^{*}
\end{array}\right)\left(\begin{array}{c}
\eta \\
\eta^{*}
\end{array}\right)=M\left(\begin{array}{c}
\eta \\
\eta^{*}
\end{array}\right),
$$


where

$$
\begin{aligned}
\eta & =\left(\begin{array}{l}
\delta_{l} \\
\delta_{r}
\end{array}\right), \\
A & =\left(\begin{array}{cc}
-\omega+2 n_{l}+i g\left(1-n_{l}\right) & -J e^{i \theta} \\
-J e^{-i \theta} & -\omega+2 n_{r}-i \gamma
\end{array}\right), \\
B & =\left(\begin{array}{cc}
n_{l}(1-i g) & 0 \\
0 & n_{r}
\end{array}\right) .
\end{aligned}
$$

Here $n_{l(r)}$ denotes the steady-state densities and $\theta$ the steadystate phase difference, obtained by solving Eqs. (6)-(9), and, as in those equations, we have set $\epsilon=0, U_{r}=U_{l}$. We see that the solutions of Eq. (A1) are of the form $\eta=\eta_{0} e^{-i v t}$, where the complex frequencies $v$ are the eigenvalues of the matrix $M$. One eigenvalue has zero decay rate and frequency and corresponds to the expected (undamped) phase mode of the condensate. The remaining eigenvalues describe the excitation spectrum in terms of the frequencies $\mathfrak{R}(\nu)$ and decay rates $\lambda=-\Im(v)$ of small fluctuations; the latter are positive for a stable steady state.
[1] T. Byrnes, N. Y. Kim, and Y. Yamamoto, Nat. Phys. 10, 803 (2014).

[2] A. Kavokin, Appl. Phys. A 89, 241 (2007).

[3] A. Smerzi, S. Fantoni, S. Giovanazzi, and S. R. Shenoy, Phys. Rev. Lett. 79, 4950 (1997).

[4] S. Raghavan, A. Smerzi, S. Fantoni, and S. R. Shenoy, Phys. Rev. A 59, 620 (1999).

[5] S. Han, R. Rouse, and J. E. Lukens, Phys. Rev. Lett. 76, 3404 (1996).

[6] Y. Yu, S. Han, X. Chu, S.-I. Chu, and Z. Wang, Science 296, 889 (2002).

[7] J. Liu, B. Wu, and Q. Niu, Phys. Rev. Lett. 90, 170404 (2003).

[8] C. Ottaviani, V. Ahufinger, R. Corbalan, and J. Mompart, Phys. Rev. A 81, 043621 (2010).

[9] D.-F. Ye, L.-B. Fu, and J. Liu, Phys. Rev. A 77, 013402 (2008).

[10] J. Kasprzak, M. Richard, S. Kundermann, A. Baas, P. Jeambrun, J. M. J. Keeling, F. M. Marchetti, M. H. Szymanśka, R. Andre, J. L. Staehli, V. Savona, P. B. Littlewood, B. Deveaud, and L. S. Dang, Nature (London) 443, 409 (2006).

[11] K. Winkler, C. Schneider, J. Fischer, A. Rahimi-Iman, M. Amthor, A. Forchel, S. Reitzenstein, S. Höfling, and M. Kamp, Appl. Phys. Lett. 102, 041101 (2013).

[12] K. G. Lagoudakis, B. Pietka, M. Wouters, R. Andre, and B. Deveaud-Pledran, Phys. Rev. Lett. 105, 120403 (2010).

[13] M. Abbarchi, A. Amo, V. G. Sala, D. D. Solnyshkov, H. Flayac, L. Ferrier, I. Sagnes, E. Galopin, A. Lemaître, G. Malpuech, and J. Bloch, Nat. Phys. 9, 275 (2013).

[14] E. A. Cerda-Méndez, D. Sarkar, D. N. Krizhanovskii, S. S. Gavrilov, K. Biermann, M. S. Skolnick, and P. V. Santos, Phys. Rev. Lett. 111, 146401 (2013).

[15] E. A. Ostrovskaya, J. Abdullaev, M. D. Fraser, A. S. Desyatnikov, and Y. S. Kivshar, Phys. Rev. Lett. 110, 170407 (2013).

[16] J. Keeling and N. G. Berloff, Phys. Rev. Lett. 100, 250401 (2008).

[17] E. A. Ostrovskaya, J. Abdullaev, A. S. Desyatnikov, M. D. Fraser, and Y. S. Kivshar, Phys. Rev. A 86, 013636 (2012).

[18] M. Wouters, Phys. Rev. B 77, 121302(R) (2008).

[19] P. R. Eastham, Phys. Rev. B 78, 035319 (2008).
[20] D. Read, Y. G. Rubo, and A. V. Kavokin, Phys. Rev. B 81, 235315 (2010).

[21] M. O. Borgh, J. Keeling, and N. G. Berloff, Phys. Rev. B 81, 235302 (2010).

[22] G. Pavlovic, G. Malpuech, and I. A. Shelykh, Phys. Rev. B 87, 125307 (2013).

[23] D. Racine and P. R. Eastham, Phys. Rev. B 90, 085308 (2014).

[24] M. Wouters, I. Carusotto, and C. Ciuti, Phys. Rev. B 77, 115340 (2008).

[25] G. Roumpos, W. H. Nitsche, S. Höfling, A. Forchel, and Y. Yamamoto, Phys. Rev. Lett. 104, 126403 (2010).

[26] I. A. Shelykh, D. D. Solnyshkov, G. Pavlovic, and G. Malpuech, Phys. Rev. B 78, 041302(R) (2008).

[27] D. Sarchi, I. Carusotto, M. Wouters, and V. Savona, Phys. Rev. B 77, 125324 (2008).

[28] B. Cao, K. W. Mahmud, and M. Hafezi, Phys. Rev. A 94, 063805 (2016).

[29] M. Galbiati, L. Ferrier, D. D. Solnyshkov, D. Tanese, E. Wertz, A. Amo, M. Abbarchi, P. Senellart, I. Sagnes, A. Lemaître, E. Galopin, G. Malpuech, and J. Bloch, Phys. Rev. Lett. 108, 126403 (2012).

[30] F. Tassone and Y. Yamamoto, Phys. Rev. B 59, 10830 (1999).

[31] A. Janot, T. Hyart, P. R. Eastham, and B. Rosenow, Phys. Rev. Lett. 111, 230403 (2013).

[32] M. Wouters and I. Carusotto, Phys. Rev. Lett. 99, 140402 (2007).

[33] M. Wouters and V. Savona, Phys. Rev. B 79, 165302 (2009).

[34] D. Read, T. C. H. Liew, Y. G. Rubo, and A. V. Kavokin, Phys. Rev. B 80, 195309 (2009).

[35] A. N. Poddubny, M. M. Glazov, and N. S. Averkiev, New J. Phys. 15, 025016 (2013).

[36] I. A. Skelykh, A. V. Kavokin, Y. G. Rubo, and G. Malpuech, Semicond. Sci. Technol. 25, 013001 (2010).

[37] J. Gillet, M. A. Garcia-March, T. Busch, and F. Sols, Phys. Rev. A 89, 023614 (2014).

[38] B. Cui, L. C. Wang, and X. X. Yi, Phys. Rev. A 82, 062105 (2010).

[39] G.-F. Wang, L.-B. Fu, and J. Liu, Phys. Rev. A 73, 013619 (2006). 